\title{
The Long-Term Effect of the Integrated Care Model on Child Morbidity in Murewa District, Zimbabwe: A Pragmatic Trial
}

\author{
Dr Maxwell Mhlanga ${ }^{1}$, Professor Midion Chidzonga ${ }^{1} \&$ Professor Clara Haruzivishe ${ }^{1}$ \\ ${ }^{1}$ College of Health Sciences, University of Zimbabwe, Harare, Zimbabwe \\ Correspondence: Maxwell Mhlanga, College of Health Sciences, University of Zimbabwe, P.O Box A178 \\ Avondale Harare, Zimbabwe. E-mail: profmaxmhlanga7@gmail.com
}

Received: June 17, 2020 Accepted: October 10, 2020 Online Published: November 17, 2020

doi:10.5539/gjhs.v12n13p138 URL: https://doi.org/10.5539/gjhs.v12n13p138

\begin{abstract}
Background: Poor access and utilisation of health care services remains a big challenge in rural communities in low to middle income countries leading to high prevalence of preventable childhood illnesses and death. Many community mobilisation models have been employed to address this. However, scientific evidence remain scanty on the long-term effects of such models to inform policy-makers on how to reduce preventable child morbidity and mortality and to provide guidance on what is pragmatic. The purpose of this study was to determine the long-term effects of the Integrated Care Model (ICM) on child morbidity and mortality in Zimbabwe.
\end{abstract}

Methods: This was a pragmatic trial that used a quasi-experimental design. The study used population based sampling to enrol villages into either intervention sites or control sites from two health centres in Murewa District. Target sampling was used to enrol children aged 0-48 months into the study. A total of 1380 children were enrolled and followed up prospectively for a period of 18 months. The disease condition that were being tracked were pneumonia, diarrhoea, fever and Malaria.

Results: We performed negative binomial logistic regression to determine the long-term effects of the intervention on child morbidity, adjusting for the number of under-fives in each village/cluster, village size, distance to the clinic and number of under-fives in each cluster. Overall, the intervention reduce the risk of general child morbidity by $83 \%[\mathrm{RR}=0.17,95 \% \mathrm{CI}(0.14-0.23)]$. The intervention reduced risk of incidence of pneumonia by $79 \%[\mathrm{RR}=0.21,95 \% \mathrm{CI}(0.10-0.45)]$, risk of incidence of diarrhoea by $80 \%$ [RR $=0.2095 \% \mathrm{CI}(0.15-0.29)]$, fever by $91 \%[\mathrm{RR}=0.09,95 \% \mathrm{CI}(0.04-0.22)]$ and malaria by $73 \%[\mathrm{RR}=0.27,95 \% \mathrm{CI}(0.14-0.51)]$. The incidence rate of childhood severe illnesses was reduced by $79 \%[\mathrm{RR}=0.24,95 \% \mathrm{CI}(0.11-0.40)]$ through the intervention.

Conclusion: This study sought to determine the long term effects of the Integrated Care Model on Child Morbidity in Murewa district, Zimbabwe. Study results revealed that indeed the ICM had a statistically significant impact on child morbidity in the long-term. Countries in low resource settings can benefit from the use of such a low-cost high impact model to reduce not only child morbidity and mortality, but also to address maternal health challenges.

Keywords: child morbidity, Integrated Care Model, positive behaviour change

\section{Introduction}

Globally, there is currently much controversy over the effectiveness of the community mobilisation models in reducing child morbidity in the long-term. Effective community mobilisation approaches are those that effectively utilise community health workers to maintain consistent and regular contact with households, developing strong partnerships with the served communities to reduce child morbidity. Currently there is still limited evidence about the effectiveness of integrated community-based approaches in reducing child morbidity. Policy makers in health are lured by community programmes that are both sustainable and effective. Close integration between community health interventions and primary health facilities are critical elements of effectiveness.

An integrated community approach empowers communities thereby increasing their self-reliance and self-confidence thereby reducing their dependence on external agents. Effective community interventions should use low-cost strategies that include home remedies, health promotion, and prevention of illnesses, early detection and treatment of childhood illnesses. One of the community mobilisation approaches that have been used in low income countries is the Care Group (CG) approach. Care Groups (CGs) have been used in countries such as Uganda, Liberia and Rwanda to promote child health and reduce child morbidity (Perry et al., 2015). 
Typical Care Group mobilisation structure heavily relies on external behaviour change communication facilitators who are paid staff and only provide services within the lifespan of a funded project usually through non-governmental organisations. A typical CG structure has managers, who have coordinators under them. Coordinators in turn have supervisors under them and the supervisors also have promoters who report to them (Perry et al., 2015). These four groups of professionals are external to the community and are paid staff.

This structure however pose challenges in terms of cost-effectiveness, replicability and sustainability due to its heavy reliance on external paid staff. An effective community model that is integrated in the health delivery system is the solution to high burden of child morbidity and mortality in low-resource settings.

Efforts have been made to integrate Care Groups in government health delivery systems and complex models involving many stakeholders such as local council and other stakeholders have been made in countries like Rwanda with good effect. A quasi-experimental study was conducted in two districts of Rwanda to determine the effectiveness of an integrated care group model on child health. This study was evaluated using mixed methods combining Knowledge, Practice and Coverage (KPC) surveys and interview (World Relief, 2015). In one of the districts, the use of Oral Rehydration Solution (ORS) for child diarrhoea rose from $22.9 \%$ at baseline to $36.1 \%$ at endline and the care seeking for pneumonia symptoms rose from $45.1 \%$ to $66.4 \%$. Community health worker home visits in the month that preceded the survey rose from $21.9 \%$ to $43.3 \%$ (World Relief, 2015).

A systematic review with meta-analysis of 24 randomised controlled trials, quasi-experimental and observational studies, estimated the effect of community-based interventions including community case management. Results revealed that community-based interventions led to significant rise in care seeking behaviour with $13 \%$ and $9 \%$ increase in care seeking for pneumonia and diarrhoea respectively (Jai, Zohra, Rehana, \& Zulfiqar, 2013). The interventions were associated with $160 \%$ increase in the use of ORS and $80 \%$ increase in use of zinc for diarrhoea (Jai et al., 2013) showing that community interventions can effectively promote child health and reduce severity of child morbidity. Effectiveness of community intervention approaches in reducing child morbidity is determined by many factors inclusive of number of interventions implemented, the quality of implementation, contextual factors and types of outcomes.

More community mobilisation models for child health have been implemented in countries like India, Bangladesh, Malawi and Nepal to effectively change care-seeking practices and to reduce morbidity by enhancing simple community interventions (Prost et al., 2014). These have shown that perinatal morbidity can be reduced in the poorest settings but the effectiveness and sustainability of delivering interventions established in low-resource settings still need to be ascertained (Prost et al., 2014).

In Nepal, success has been achieved through health partnerships. Greater community involvement in the health promotion activities resulted in greater impact and more sustainable gains of a community intervention (Prost et al., 2014). In Malawi, community mobilisation approaches lowered the neonatal morbidity rate by $22 \%$ in intervention clusters than control clusters ( $\mathrm{OR}=0.78,95 \%$ CI 0.60-1.01) (Colbourn et al., 2015).

A cluster randomised control trial by on targeted networks revealed that community networks can improve behaviour change (Perry, Rassekh, Gupta, Wilhelm, \& Freeman, 2017). An intervention in this study called targeted nominated friends increased adoption of nutritional intervention by $12.2 \%$ compared with random targeting (95\% CI 6.9-17.9). Effective community intervention models utilise recommended approaches using local resources that are contextual, aligning with the community's knowledge, skills and interests. In an effective model, transformation is contagious, spreading to other households via community health workers. Effectiveness is not restricted only to specific interventions but also the delivery approaches at community level (Alba et al., 2010).

Effective community mobilisation approaches are the conduits for decentralisation of existing health services and programmes as they target total community coverage in a geographically defined population resulting in effective health communication with individuals, families and communities. Effective social mobilisation for community involvement in health and provision of healthcare at household level (both preventive and curative services) is the ultimate outcome.

Social networks provide a vehicle through which health programming can affect those with close social ties to the targeted beneficiaries. The ACCESS programme in rural Tanzania sought to improve access to prompt malaria treatment. The ACCESS study realized a decline in childhood illness by $34 \%$ from 4.9 to 3.2 average cases per child year amongst children under 5 (Mazumder et al., 2014).

A cluster-randomised controlled trial in India on the impact of inequities in health indicators assessed the effectiveness of implementing the Integrated Management of Newborn and Childhood Illness (IMNCI) 
programme in Haryana in India. Findings of the trial revealed that the IMNCI strategy resulted in lower infant morbidity and improved infant care practices (McGlynn, Wilk, Luginaah, Ryan, \& Thind, 2015). Multiple linear regression analysis was used to determine inequity gradients in neonatal morbidity, care practices and care seeking and to assess the differences in these gradients between intervention and control clusters. The intervention resulted in a larger effect on breastfeeding within one hour of birth in poorer households (difference in inequity gradients $3.0 \%$, CI 1.5 to $4.5, \mathrm{P}<0.001$ ), in lower caste and minorities, and in infants of mothers with poor education. The intervention reduced gender inequity in care seeking for neonatal illness from health centres (difference in inequity gradients $9.3 \%$, CI 0.4 to $18.2, \mathrm{P}=0.042$ ) (McGlynn, et al., 2015).

A study in Ghana investigated whether increased use of recommended maternal healthcare (MHC) is a determinant of immunisation and appropriate care for children with fever and diarrhoea using demographic health survey data (Harkins, Drasbek, Arroyo, \& McQuestion, 2008). Increased utilisation of services increased the odds of immunisation [poor use: odds ratio $(\mathrm{OR})=0.54,95 \%$ confidence interval $(\mathrm{CI}): 0.42-0.69$; best use: $\mathrm{OR}=1.29$, 95\% CI: 1.01-1.67]20. MCH utilisation also increased odds of appropriate care for fever (poor use: $\mathrm{OR}=0.55$, 95\% CI: $0.35-0.88$; best use: $\mathrm{OR}=1.72,95 \%$ CI: $1.17-2.52$ ) and diarrhoea (poor use: $\mathrm{OR}=0.63,95 \% \mathrm{CI}$ : 0.43-0.93) (Harkins, et al., 2008).

There is suggestive evidence from these studies that community mobilisation approaches effectively reduce child morbidity. The only challenge in most of the current evidence is that most of the approaches showed effectiveness at pilot stages and have not been tested for their long term effects in a pragmatic way when other confounding factors come into play in a real day to day operating environment.

Assessing effectiveness of community health interventions in real life situation is top priority in that it improves quality of decision making, enhance efficiency and enlightens policy makers on why some approaches works and others fail. Innovations have died a natural death since they could not be integrated into government structures due to various reasons that include lack of ownership by government, cost-effectiveness and sustainability. The reviewed studies did not provide information on the cost versus benefits of the community interventions. A cost-benefit analysis of the community interventions in reducing child morbidity is critical if policy makers are to consider implementing such interventions at national level.

The lack of comparative studies that assess effectiveness of community health interventions make it difficult to know whether novel and innovative mobilisation approaches are more effective in reducing child morbidity if integrated in the healthcare delivery system or conventional strategies used in developing countries by the respective governments are the most effective models. This study is one of the very few studies in Zimbabwe to directly compare and evaluate the effectiveness of an integrated innovative community mobilisation approach in reducing child morbidity against the conventional mobilisation approach in the long term.

The community mobilisation model was first tested in 2017 in Mashonaland East using a cluster randomised controlled trial that involved 4 districts. This innovative Integrated Care Model (ICM) was compared to the conventional mobilization system over a follow-up period of twelve months and the effect of the intervention was assessed. The intervention reduced child morbidity by $80 \%$ with a statistically significant impact $(\mathrm{p}<0.001)$ on all maternal knowledge and care practice indicators that the study measured. After a latent period of one year, we then went back to assess the long-term effect of the intervention in the same region.

Figure 1 below is a schematic representation of the Integrated Care Model versus the rural community health system in Zimbabwe. The basic difference between villages in the intervention and control arm is the way health education, health promotion and monitoring is conducted, with the intervention villages having an extended team of community health volunteers to total reach all households as opposed to the existence of one village health worker only per village in the conventional health system. 


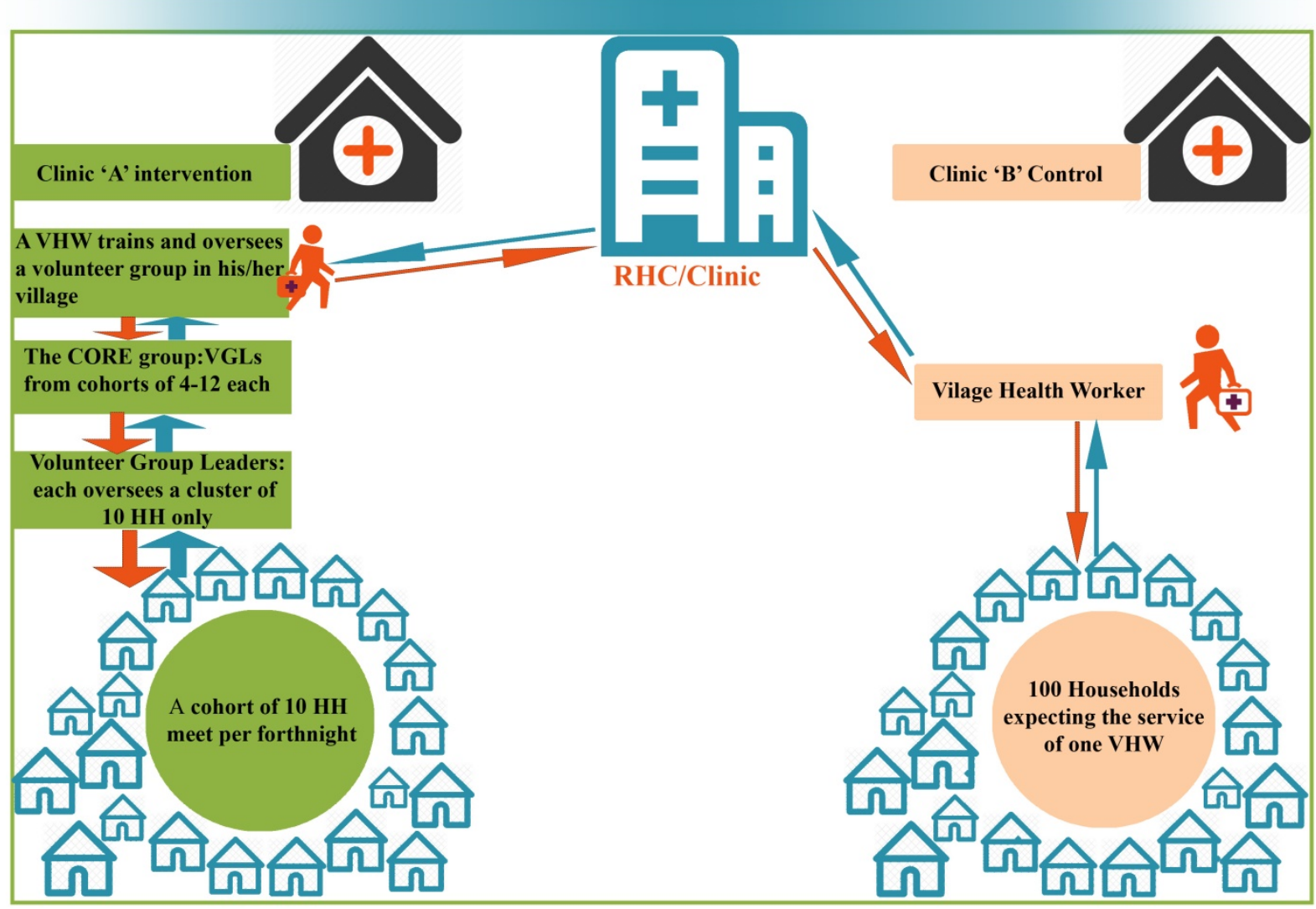

Figure 1. The Integrated Care Model and the conventional health system

\section{Methods}

This was a pragmatic trial and a quasi-experimental designs was used. The study used population based sampling to enrol villages into either intervention sites or control sites. Target sampling was used to enroll children aged 0-48 months into the study. We also examined differences across two sub-groups 0-24 months and 25-48 months.

\subsection{Description of the Intervention}

The intervention villages had an extended structure of community volunteer health educators in form of Volunteer Group leaders (VGLs) in addition to the conventional Village Health Worker (VHW). The VHW used an integrated community maternal and child health promotion manual to train a cohort of 10 VGLs selected from sub-clusters of households in each intervention village. Each Volunteer Group Leader (VGL) was a caregiver of at least one child who is 0 to 48 months old and was selected by the members from a cohort (sub-clusters) of 10 households in their locality, whom they represented in the CG. This was done with the full support and buy-in of local traditional leadership (Headmen).Each VHW in the intervention group received a five days' training on how to facilitate the sessions with VGLs using participatory approaches and how to provide supportive visits. The VGLs in return also cascaded the same sessions to the sub-clusters of households and the sessions were 1-2 hours long held once every fortnight. The local health centre nurse received training on how to monitor and coordinate the sessions and is overall in charge of the community health interventions and directly supervises the activities.

The nurse had an overall responsibility of giving feedback to the community and updates on new guidelines on childhood sickness management hence there was a continuous two-way communication between the communities in the clinic's catchment area and the health centre mediated by the VHWs. A village in Zimbabwe has an average size of 150 households hence the average number of volunteer members in each Core Group was 10.

In the sessions, the participants shared their experiences and challenges in pregnancy, delivery, post-delivery and childcare and these were recorded respectively. Participatory approaches such as drama, songs, testimonials, case studies, phot galleries, simulations, demonstrations, community mapping, story-telling, problem tree analysis and many more were used to provide edutainment. 
Principles of the 'open space technology' were incorporated in sessions to allow for full, active participation of all members and enhance accountability in the matters relating to their own health. VGLs primarily targeted care-givers of children 0-59 months and pregnant women in their clusters of 10 households though other people were free to join sessions. The VGLs conducted at least two home visits each month to monitor health practices and behaviours and also provide support and individualized health education in targeted households.

Community-integrated management of childhood illness topics included good health care, community prevention and management of diarrhoea, good childcare, expanded program on immunisation, importance of regular growth monitoring, prevention and management of malaria. Other topics were on prevention of pneumonia and danger signs, when to seek healthcare services at the health facility and importance of community health workers in child health.

\subsection{Description of Control}

The villages that were not in the previous trial, served as the study control. The VHWs in such villages continued their day to day routine activities according to the guidelines of the Ministry of Health and Child Care and reported to the same clinic. The Ministry of Health guidelines stipulate that VHWs should be able to carry out all the 14 elements of the Primary Health approach at village level and report their activities monthly to their nearest clinic.

\section{Results and Analysis}

\subsection{Descriptive Statistics}

Table 1 below summarises the demographic characteristics of the study sites. A total of 30 villages (Clusters) constituted the catchment area for the study, and a total of 1380 under-fives from these villages participated in the study.

Table 1. Demographic characteristics of project sites

\begin{tabular}{llll}
\hline Variable & Murewa Health Centre & Macheke Health centre & Overall \\
\hline Number of villages (Clusters) & $17(56.7 \%)$ & $13(43.3 \%)$ & $30(100.0 \%)$ \\
Number of villages in the Intervention arm & $9(60.0 \%)$ & $6(40.0 \%)$ & $15(100.0 \%)$ \\
Number of villages in the control arm & $8(53.3 \%)$ & $7(46.7 \%)$ & $15(100.0 \%$ \\
Mean Distance $(\mathrm{km})$ to the clinic & $9.5(\mathrm{SD}=7.3)$ & $11.5(\mathrm{SD}=7.0)$ & $10.5(\mathrm{SD}=7.1)$ \\
Mean Village size $(\mathrm{HH})$ & $108(\mathrm{SD}=25)$ & $129(\mathrm{SD}=53)$ & $119(\mathrm{SD}=42)$ \\
Total number of U5s per site & $808(58.6 \%)$ & $572(41.4 \%)$ & $1380(100.0 \%)$ \\
Mean Number of children below 5 years & $42(\mathrm{SD}=12)$ & $50(\mathrm{SD}=21)$ & $46(\mathrm{SD}=18)$ \\
\hline
\end{tabular}

Table 2. Descriptive statistics for childhood illnesses

\begin{tabular}{lll}
\hline Variable(Cases) & Intervention Mean (SD) & Control Mean (SD) \\
\hline Pneumonia & $22.8(\mathrm{SD}=19.8)$ & $105.6(\mathrm{SD}=82.4)$ \\
Diarrhoea & $19.6(\mathrm{SD}=12.7)$ & $74.0(\mathrm{SD}=32.9)$ \\
Fever & $4.7(\mathrm{SD}=5.8)$ & $40.9(\mathrm{SD}=20.7)$ \\
Malaria & $0.9(\mathrm{SD}=0.5)$ & $3.4(\mathrm{SD}=1.4)$ \\
Overall & $48(\mathrm{SD}=24.2)$ & $223.9(\mathrm{SD}=96.2)$ \\
Severe cases & $9.8(\mathrm{SD}=10.2)$ & $40.7(\mathrm{SD}=19.6)$ \\
\hline
\end{tabular}

\subsection{Outcomes and Estimations}

We performed negative binomial logistic regression to determine the long-term effects of the intervention on child morbidity, adjusting for the number of under-fives in each village/cluster, village size, distance to the clinic and number of under-fives in each cluster. Overall, the intervention reduce the risk of general child morbidity by $83 \%$. The intervention reduced risk of incidence of pneumonia by $79 \%$, risk of incidence of diarrhoea by $80 \%$, fever by $91 \%$ and malaria by $73 \%$. The incidence rate of childhood severe illnesses was reduced by $79 \%$ through the intervention. Table 3 below shows the overall results and the results by disease condition. 
Table 3. Outcomes and estimations

\begin{tabular}{lccc}
\hline Reference arm: Control arm & IRR (Incidence Rate Ratio) & p-value & 95\% Confidence Interval \\
\hline Pneumonia & 0.21 & $<0.001$ & $0.10-0.45$ \\
Diarrhoea & 0.20 & $<0.001$ & $0.15-0.29$ \\
Fever & 0.09 & $<0.001$ & $0.04-0.22$ \\
Malaria & 0.27 & $<0.001$ & $0.14-0.51$ \\
Overall & 0.17 & $<0.001$ & $0.14-0.23$ \\
Severe cases & 0.21 & $<0.001$ & $0.11-0.40$ \\
\hline
\end{tabular}

\section{Discussion}

The study results have indicated that the intervention had a statistically significant long-effect on child morbidity and mortality for all disease conditions that were assessed, and the result effect was even higher compared to the baseline effect when assessed a year after the intervention has been set in motion and allowed to roll-on in a pragmatic situation.

Similar studies on child morbidity have revealed that effective community intervention approaches can reduce child morbidity. A quasi-experimental study $(\mathrm{N}=300)$ conducted in San Luis reduced the incidence of diarrhoeal diseases among children aged 0-59 months by $18 \%$ between survey rounds (Hashi, Kumie, \& Gasana, 2017).

High immunisation coverage and the introduction of the pneumococcal vaccine to curb the morbidity and mortality due to pneumonia has helped in reducing pneumonia cases in Zimbabwe. However, although pneumonia can both be prevented and treated with low-cost interventions, it remains the leading infectious cause of death in children below five in Zimbabwe and other Sub-Saharan countries. Risk for pneumonia in children is exacerbated by malnutrition, low birth weight, the lack of exclusive breastfeeding for the first 6 months of life, HIV infection or exposure, indoor air pollution (caused by smoking or cooking with solid fuels) and airway inflammation. These factors are preventable and the community education sessions in the intervention villages addressed these in care-givers and this could also explain the significant variation in cases between villages with the intervention and those without. Target specific and focused messaging and awareness raising yields measurable impact in child morbidity.

A study in Ethiopia that sought to promote hand-washing with soap and WASH educational intervention to reduce childhood diarrhoea incidence using a community-based cluster randomised trial reported an Incidence Rate Ratio (IRR) of $0.6595 \%$ C.I (0.57-0.73) and this meant the intervention reduced diarrhoeal cases by $35 \%$ (Rudan et al., 2013). This study reported a higher effect on diarrhoea between the control villages and the intervention. Causes of childhood diarrhoea are largely preventable and these include lack of EBF for the first 6 months of life, using infant formula/unclean water, improper storage of food in warm environments, poor hand hygiene and poor disposal of faecal matter. The community manual use in the ICM model addresses these fully and the model has a strong monitoring system of behaviours at household level. The GAPPD framework informs country policies and programme practices relating to the protection of children from diarrhoea through exclusive breastfeeding for 6 months, complementary feeding and vitamin A supplementation (Van den, Brown, Hoekstra, Christie, \& Cochi, 2008). Diarrhoea mortality is highest between 6 and 11 months of age and is responsible for 19 percent of all child deaths and continued breastfeeding with proper introduction of complementary feeds can save lives. The GAPPD framework recommends prevention of diarrhoea through provision of vaccines for rotavirus and measles, hand-washing with soap and improving access to safe drinking water and sanitation. Anecdotal data from our study showed that diarrhoea cases were higher in specific seasons and periods of the year and it is necessary to intensify mobilisation and awareness campaigns during specific time periods so as to ensure consistent, positive child health outcomes and adoption of good caring practices.

This study showed a significant long-term effect in Malaria and fever reduction among children below five years. Similarly, the ACCESS community programme implemented in Tanzania to prevent and manage malaria in rural communities reported that fever incidence rates in children under the age of 5 years declined by $34 \%$ from 4.9 to 3.2 average cases per child year. Convulsions, indicator of severe malaria morbidity in children, decreased by $46 \%$, from 4263 and 2320 cases for every, 100000 children per year 18 .

The study intervention enhanced malaria prevention strategies to reduce the mosquito population especially by increasing the coverage of Long-lasting insecticide treated-Nets (LITNs). This was made possible through 
behaviour change communication focused on the correct and consistent use of nets coupled with door-to-door behaviour monitoring through home visits by community volunteers. The initial census conducted in each village before the establishment of groups was instrumental in establishing an accurate village population disaggregated by age. This was important in ensuring $100 \%$ coverage in net distribution and fostering the 'community effect'. When LITNs are used by the majority of residents in a community, they reduce vector population and provide protection to the whole community, even those community members who are not sleeping under a net. This is what is known as the 'community effect'.

The study showed a very significant long-term effect. A few limitations were also faced. The age range of 0-59 months is quite wide, housing different sub-groups of children such as infants, young children and neonates whose vulnerability to childhood illnesses differ by age categories. However, care-giver knowledge, attitude and care practices are not affected by the age of the child and the developed model was targeting these to improve health outcomes for children of all age groups who are below five years of age.

This study recommends that governments in developing countries integrate community health mobilisation and social behaviour change communication interventions into a single main-streamed approach under their respective community health system strategies. This will reduce costs and prevent the duplication of resources and efforts for the same cause and provides a sustainable and effective way of delivering quality health care at community level. The fragmented approach that is currently used in most countries is costly and pays fewer dividends.

Governments in developing countries should ensure that community health workers are fully equipped with kits that allow them to treat childhood illness at very early stages. Since early treatment is cheaper than referral, community health workers should be fully capacitated and effectively monitored in their screening and management of childhood illnesses. All community health workers should have community health manuals that they use continuously to refer to for consistency in information disseminated and for them to also cover more content on Integrated Management of Newborn and Childhood illnesses.

\section{Competing Interests Statement}

The authors declare that there are no competing or potential conflicts of interest.

\section{References}

Alba, S., Dillip, A., Hetzel, M. W., Mayumana, I., Mshana, C., Makemba, A., ... \& Lengeler, C. (2010). Improvements in access to malaria treatment in Tanzania following community, retail sector and health facility interventions -- a user perspective. Malaria Journal, 15, 9-163 https://doi.org/10.1186/1475-2875-9-163

Colbourn, T., Nambiar, B., Bondo, A., Makwenda, C., Tsetekani, E., Makonda-Ridley, A., ... \& Christakis, N. A. (2015). Social network targeting to maximise population behaviour change: a cluster randomised controlled trial. The Lancet, 386, 145-153 https://doi.org/10.1016/S0140-6736(15)60095-2

Harkins, T., Drasbek, C., Arroyo, J., \& McQuestion, M. (2008). The health benefits of social mobilisation: experiences with community-based Integrated Management of Childhood Illness in Chao, Peru and San Luis, Honduras. Promot Educ, 15, 15-20. https://doi.org/10.1177/1025382308090340

Hashi, A., Kumie, A., \& Gasana, J. (2017). Hand washing with soap and WASH educational intervention reduces under-five childhood diarrhoea incidence in Jigjiga District, Eastern Ethiopia: A community-based cluster randomised controlled trial. Prev Med Rep, 6, 361-368. https://doi.org/10.1016/j.pmedr.2017.04.011

Jai, D. K., Zohra, S. L., Rehana, A. S., \& Zulfiqar, A. B. (2013). Effect of community- based interventions on childhood diarrhea and pneumonia: uptake of treatment modalities and impact on mortality. BMC Public Health, 13, 3-29. https://doi.org/10.1186/1471-2458-13-S3-S29

Mazumder, S., Taneja, S., Bahl, R., Mohan, P., Strand, T. A., Sommerfelt, H., ... \& Bhandari, N. (2014). Integrated Management of Neonatal and Childhood Illness Evaluation Study Group.Effect of implementation of integrated management of neonatal and childhood illness programme on treatment seeking practices for morbidities in infants: cluster randomised trial. BMJ, 349, 1-15. https://doi.org/10.1136/bmj.g4988

McGlynn, N., Wilk, P., Luginaah, I., Ryan, B. L., \& Thind, A. (2015). Increased use of recommended maternal healthcare as a determinant of immunisation and appropriate care for fever and diarrhoea in Ghana: an analysis pooling three demographic and health surveys. Health Policy Plan, 30(7), 895-905. https://doi.org/10.1093/heapol/czu090

Perry, H. B., Rassekh, B. M., Gupta, S., Wilhelm, J., \& Freeman, P. A. (2017). Comprehensive review of the 
evidence regarding the effectiveness of community-based primary healthcare in improving maternal, neonatal and child health. J Glob Health,7(1), 010901 https://doi.org/10.7189/jogh.07.010901

Perry, H., Morrow, M., Borger, S., Weiss, J., DeCoster, M., \& Davis, T. (2015). Care Groups I: an innovative community-based strategy for improving maternal, neonatal, and child health in resource-constrained settings. Glob Health Sci Pract, 3(3), 358-36. https://doi.org/10.9745/GHSP-D-15-00051

Prost, A., Colbourn, T., Seward, N., Copas, A., Houweling, T. A., Fottrell, E., ... \& Costello, A. (2014). Women's groups practicing participatory learning and action to improve maternal and newborn health in low-resource settings: a systematic review and meta-analysis. The Lancet, 381, 1736-1746. https://doi.org/10.1016/S0140-6736(13)60685-6

Rudan, I., O'Brien, K. L., Nair, H., Liu, L., Theodoratou, E., Qazi, S., .. \& Campbell, H. (2013). Child Health Epidemiology Reference Group (CHERG).Epidemiology and etiology of childhood pneumonia in 2010: estimates of incidence, severe morbidity, mortality, underlying risk factors and causative pathogens for 192 countries. J Glob Health, 3(1), 1-42.

Van den, E., Brown, D. W., Hoekstra, E. J., Christie, A., \& Cochi, L. (2008). Measles Mortality Reduction Contributes Substantially to Reduction of All Cause Mortality Among Children Less Than Five Years of Age, 1990-2008. The Journal of Infectious Diseases, 204(1), S18-S23. https://doi.org/10.1093/infdis/jir081

World Relief. (2015). Innovating for child health: An evaluation of an integrated care group model in Rwanda (pp. 1-12). Final report. United States Agents of International Development.

\section{Copyrights}

Copyright for this article is retained by the author(s), with first publication rights granted to the journal.

This is an open-access article distributed under the terms and conditions of the Creative Commons Attribution license (http://creativecommons.org/licenses/by/4.0/). 